Supporting Information

\title{
Total Synthesis of the Cyclopeptide Alkaloid Paliurine E. Insights into Macrocyclization by Ene-enamide RCM
}

\author{
Mathieu Toumi, François Couty and Gwilherm Evano* \\ Institut Lavoisier de Versailles, UMR CNRS 8180 \\ Université de Versailles Saint-Quentin en Yvelines \\ 45, avenue des Etats-Unis \\ 78035 Versailles Cedex \\ France
}

Paliurine E Spectral Data Compared to Reported Data...................................S2

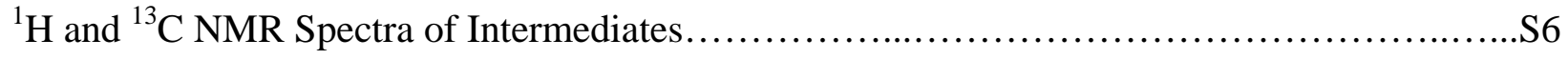

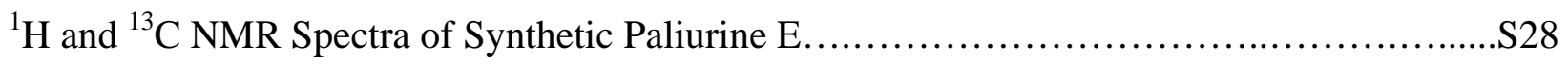




\section{Supporting Information}

\section{Paliurine E Spectral Data Compared to Reported Data}

${ }^{1} \mathbf{H}$ NMR (Synthetic at $300 \mathrm{MHz}$; Natural at $400 \mathrm{MHz}$ )

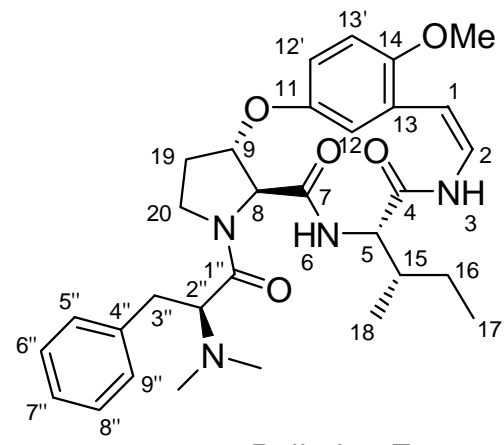

Paliurine $\mathrm{E}$

\begin{tabular}{|c|c|c|c|c|c|}
\hline & & Synthetic & & Natural & \\
\hline Position & $\delta$ & multiplicity & $\delta$ & multiplicity & $\Delta \delta$ \\
\hline $1(1 \mathrm{H})$ & 5.91 & d, $J=9.1 \mathrm{~Hz}$ & 5.90 & d, $J=9.0 \mathrm{~Hz}$ & 0.01 \\
\hline $2(1 \mathrm{H})$ & 6.94 & $\mathrm{dd}, J=9.2,11.3 \mathrm{~Hz}$ & 6.92 & dd, $J=9.0,11.3 \mathrm{~Hz}$ & 0.02 \\
\hline $3(1 \mathrm{H})$ & 8.43 & $\mathrm{~d}, J=11.4 \mathrm{~Hz}$ & 8.41 & d, $J=11.3 \mathrm{~Hz}$ & 0.02 \\
\hline $5(1 \mathrm{H})$ & 4.24 & app. t, $J=4.2 \mathrm{~Hz}$ & 4.22 & $\mathrm{dd}, J=4.4,4.3 \mathrm{~Hz}$ & 0.02 \\
\hline $6(1 \mathrm{H})$ & 7.40 & d, $J=4.5 \mathrm{~Hz}$ & 7.38 & d, $J=4.3 \mathrm{~Hz}$ & 0.02 \\
\hline $8(1 \mathrm{H})$ & 4.44 & $\mathrm{~d}, J=3.0 \mathrm{~Hz}$ & 4.43 & $\mathrm{~d}, J=3.0 \mathrm{~Hz}$ & 0.01 \\
\hline $9(1 \mathrm{H})$ & 5.41 & $\mathrm{dt}, J=7.2,3.0 \mathrm{~Hz}$ & 5.40 & $\mathrm{dt}, J=7.2,3.0 \mathrm{~Hz}$ & 0.01 \\
\hline $12(1 \mathrm{H})$ & 6.68 & d, $J=2.9 \mathrm{~Hz}$ & 6.67 & d, $J=3.0 \mathrm{~Hz}$ & 0.01 \\
\hline $12^{\prime}(1 \mathrm{H})$ & 6.79 & $\mathrm{dd}, J=9.0,2.9 \mathrm{~Hz}$ & 6.78 & $\mathrm{dd}, J=9.0,3.0 \mathrm{~Hz}$ & 0.01 \\
\hline $13^{\prime}(1 \mathrm{H})$ & 6.88 & $\mathrm{~d}, J=9.0 \mathrm{~Hz}$ & 6.85 & d, $J=9.0 \mathrm{~Hz}$ & 0.03 \\
\hline $15(1 \mathrm{H})$ & $2.03-2.21$ & $\mathrm{~m}$ & 2.05 & $\mathrm{~m}$ & $\mathrm{NA}^{\mathrm{a}}$ \\
\hline
\end{tabular}




\begin{tabular}{|c|c|c|c|c|c|}
\hline $16(2 \mathrm{H})$ & $1.13-1.48$ & $\mathrm{~m}$ & $\begin{array}{c}1.16 \text { and } \\
1.42\end{array}$ & $\mathrm{~m}$ & $\mathrm{NA}^{\mathrm{a}}$ \\
\hline $17(3 \mathrm{H})$ & 0.92 & $\mathrm{t}, J=7.3 \mathrm{~Hz}$ & 0.91 & $\mathrm{t}, J=7.3 \mathrm{~Hz}$ & $\mathrm{NA}^{\mathrm{a}}$ \\
\hline $18(3 \mathrm{H})$ & 1.01 & d, $J=7.0 \mathrm{~Hz}$ & 0.99 & d, $J=7.0 \mathrm{~Hz}$ & 0.02 \\
\hline $19 \alpha(1 \mathrm{H})$ & $2.03-2.21$ & $\mathrm{~m}$ & 2.14 & $\mathrm{~m}$ & $\mathrm{NA}^{\mathrm{a}}$ \\
\hline $19 \beta(1 \mathrm{H})$ & $2.36-2.39$ & $\mathrm{~m}$ & 2.40 & $\mathrm{~m}$ & $\mathrm{NA}^{\mathrm{a}}$ \\
\hline $20 \alpha(1 \mathrm{H})$ & 4.00 & ddd, $J=10.9,8.5,2.7 \mathrm{~Hz}$ & 4.00 & ddd, $J=10.8,8.3,2.8 \mathrm{~Hz}$ & 0.00 \\
\hline $20 \beta(1 \mathrm{H})$ & 2.86 & $\mathrm{dt}, J=4.2,10.7 \mathrm{~Hz}$ & 2.86 & $\mathrm{dt}, J=5.9,10.7 \mathrm{~Hz}$ & 0.00 \\
\hline 2" (1H) & 3.44 & $\mathrm{dd}, J=10.3,3.5 \mathrm{~Hz}$ & 3.42 & $\mathrm{dd}, J=10.3,3.4 \mathrm{~Hz}$ & 0.02 \\
\hline 3" (1H) & 3.06 & $\mathrm{dd}, J=13.0,10.6 \mathrm{~Hz}$ & 3.06 & $\mathrm{dd}, J=13.0,10.3 \mathrm{~Hz}$ & 0.00 \\
\hline 3" (1H) & 2.92 & dd, $J=13.0,3.3 \mathrm{~Hz}$ & 2.91 & $\mathrm{dd}, J=13.0,3.4 \mathrm{~Hz}$ & 0.01 \\
\hline $5 "+9 "(2 \mathrm{H})$ & 7.06 & $\mathrm{dd}, J=7.7,1.7 \mathrm{~Hz}$ & 7.04 & $\mathrm{dd}, J=7.2,1.2 \mathrm{~Hz}$ & 0.02 \\
\hline $6 "+8 "(2 \mathrm{H})$ & $7.15-7.24$ & $\mathrm{~m}$ & 7.17 & $\mathrm{~m}$ & $\mathrm{NA}^{\mathrm{a}}$ \\
\hline 7" (1H) & $7.15-7.24$ & $\mathrm{~m}$ & 7.17 & $\mathrm{~m}$ & $\mathrm{NA}^{\mathrm{a}}$ \\
\hline OMe (3H) & 3.79 & s & 3.78 & $\mathrm{~s}$ & 0.01 \\
\hline $\mathrm{NMe}_{2}(6 \mathrm{H})$ & 2.40 & $\mathrm{~s}$ & 2.39 & $\mathrm{~s}$ & 0.01 \\
\hline
\end{tabular}

${ }^{\mathrm{a}} \mathrm{NA}=$ not available 
${ }^{13}$ C NMR (Synthetic at $75 \mathrm{MHz}$; Natural at $100 \mathrm{MHz}$ )

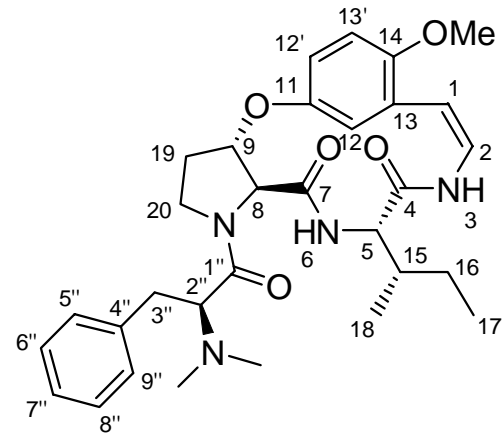

Paliurine E

\begin{tabular}{|c|c|c|c|}
\hline & Synthetic & Natural & \\
\hline Position & $\delta$ & $\delta$ & $\Delta \delta$ \\
\hline 1 & 106.7 & 106.6 & 0.1 \\
\hline 2 & 121.7 & 121.5 & 0.2 \\
\hline 4 & 167.4 & 167.2 & 0.2 \\
\hline 5 & 60.5 & 60.3 & 0.2 \\
\hline 7 & 170.3 & 170.2 & 0.1 \\
\hline 8 & 64.6 & 64.5 & 0.1 \\
\hline 9 & 76.9 & 76.8 & 0.1 \\
\hline 11 & 151.2 & 151.1 & 0.1 \\
\hline 12 & 111.4 & 111.3 & 0.1 \\
\hline $12^{\prime}$ & 117.6 & 117.5 & 0.1 \\
\hline 13 & 124.5 & 124.4 & 0.1 \\
\hline $13^{\prime}$ & 113.9 & 113.8 & 0.1 \\
\hline 14 & 151.6 & 151.4 & 0.2 \\
\hline 15 & 35.7 & 35.6 & 0.1 \\
\hline 16 & 24.8 & 24.7 & 0.1 \\
\hline 17 & 12.1 & 11.8 & 0.3 \\
\hline 18 & 16.4 & 16.3 & 0.1 \\
\hline
\end{tabular}




\begin{tabular}{|c|c|c|c|}
\hline 19 & 32.4 & 32.3 & 0.1 \\
\hline 20 & 46.0 & 45.8 & 0.2 \\
\hline $1 "$ & 171.3 & 171.2 & 0.1 \\
\hline $2 "$ & 68.2 & 68.0 & 0.2 \\
\hline $3 "$ & 32.5 & 32.2 & 0.3 \\
\hline $4 "$ & 138.3 & 138.2 & 0.1 \\
\hline $5^{\prime \prime}+9 "$ & 129.1 & 128.9 & 0.2 \\
\hline $6^{\prime \prime}+8 "$ & 128.7 & 128.6 & 0.1 \\
\hline $7 "$ & 126.7 & 126.5 & 0.2 \\
\hline $\mathrm{OMe}^{\prime \prime}$ & 56.2 & 56.0 & 0.2 \\
\hline $\mathrm{NMe}_{2}$ & 42.1 & 41.9 & 0.2 \\
\hline
\end{tabular}


Supporting Information

\section{${ }^{1} \mathrm{H}$ and ${ }^{13} \mathrm{C}$ NMR spectra of synthetic intermediates}



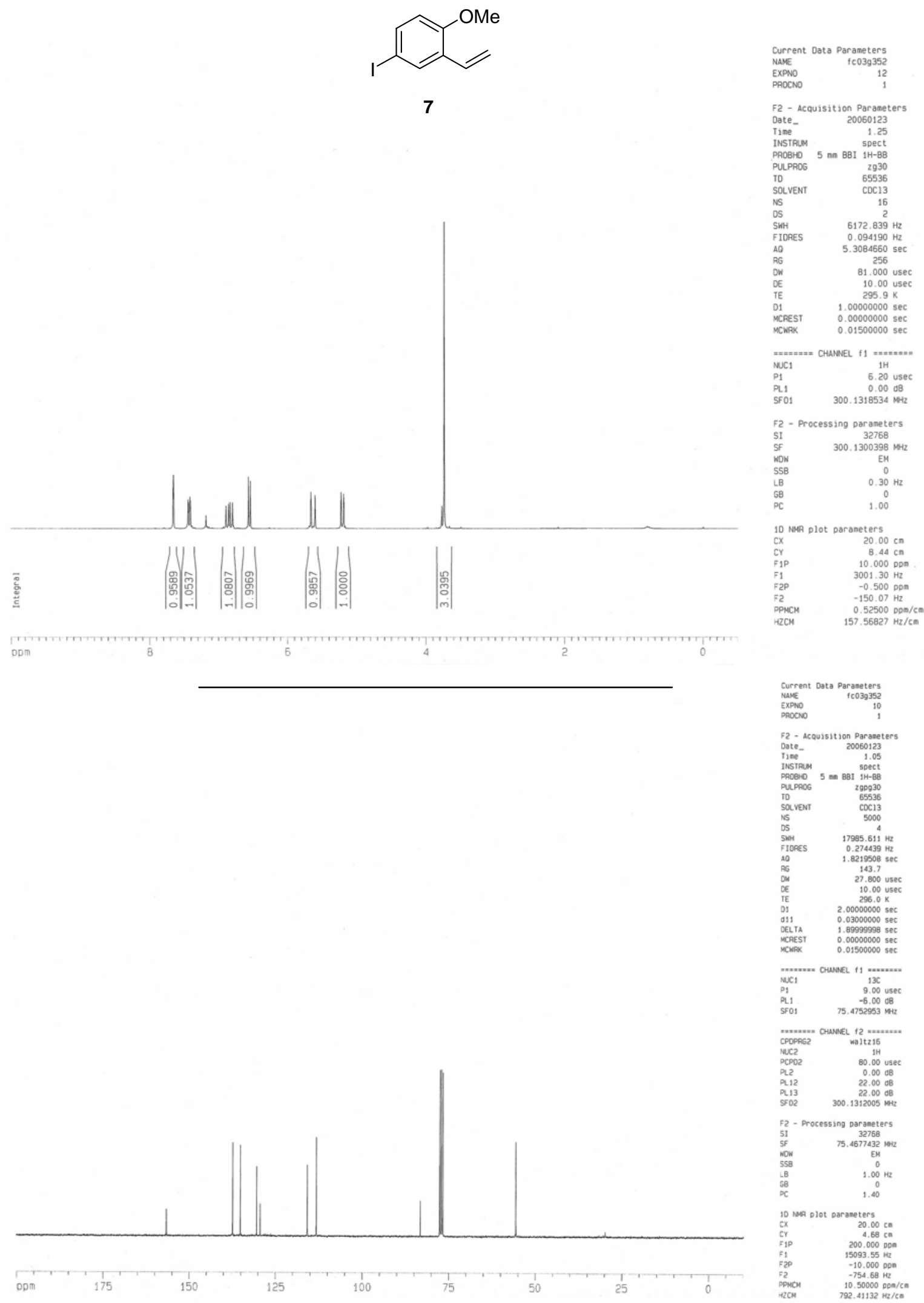


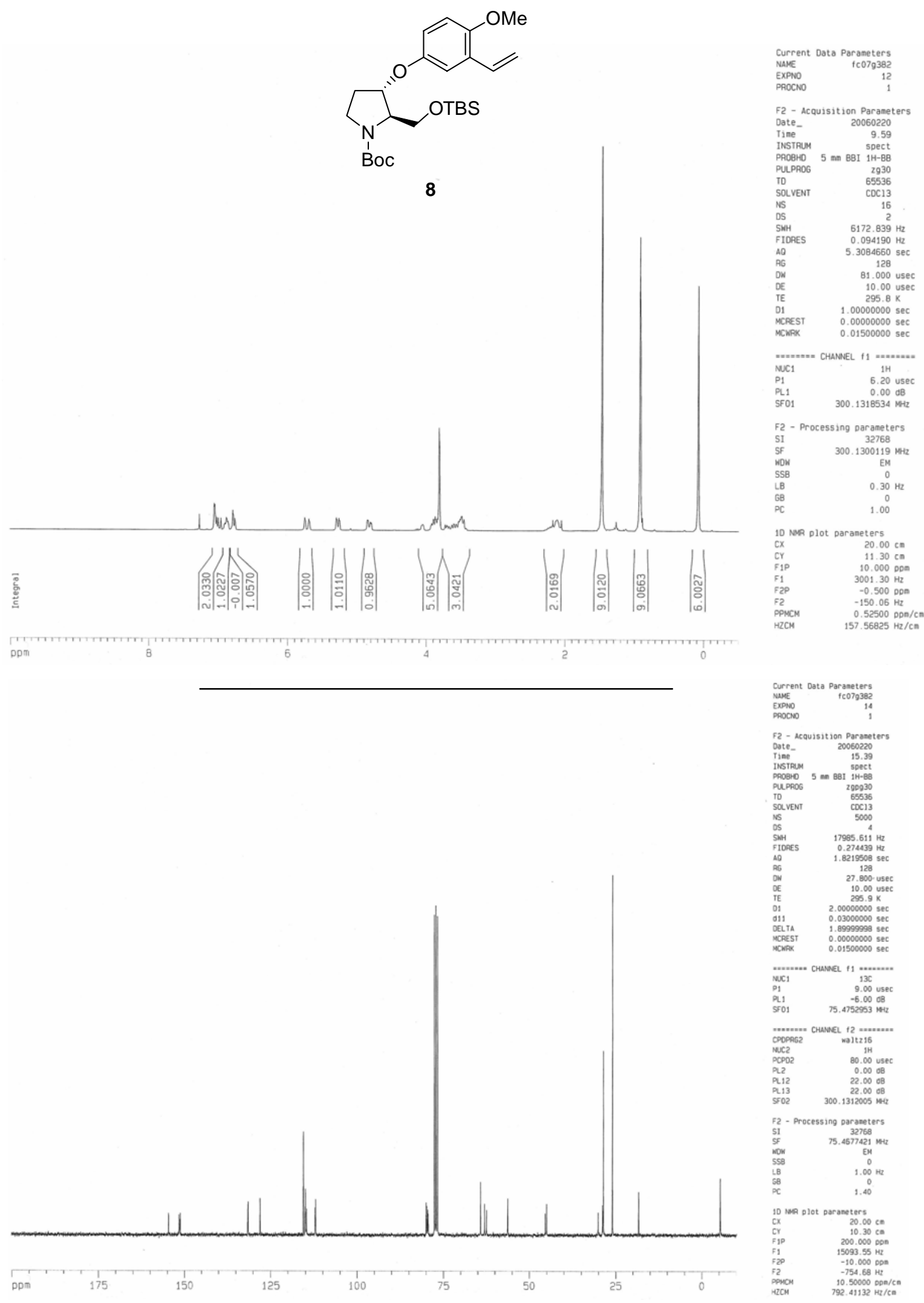



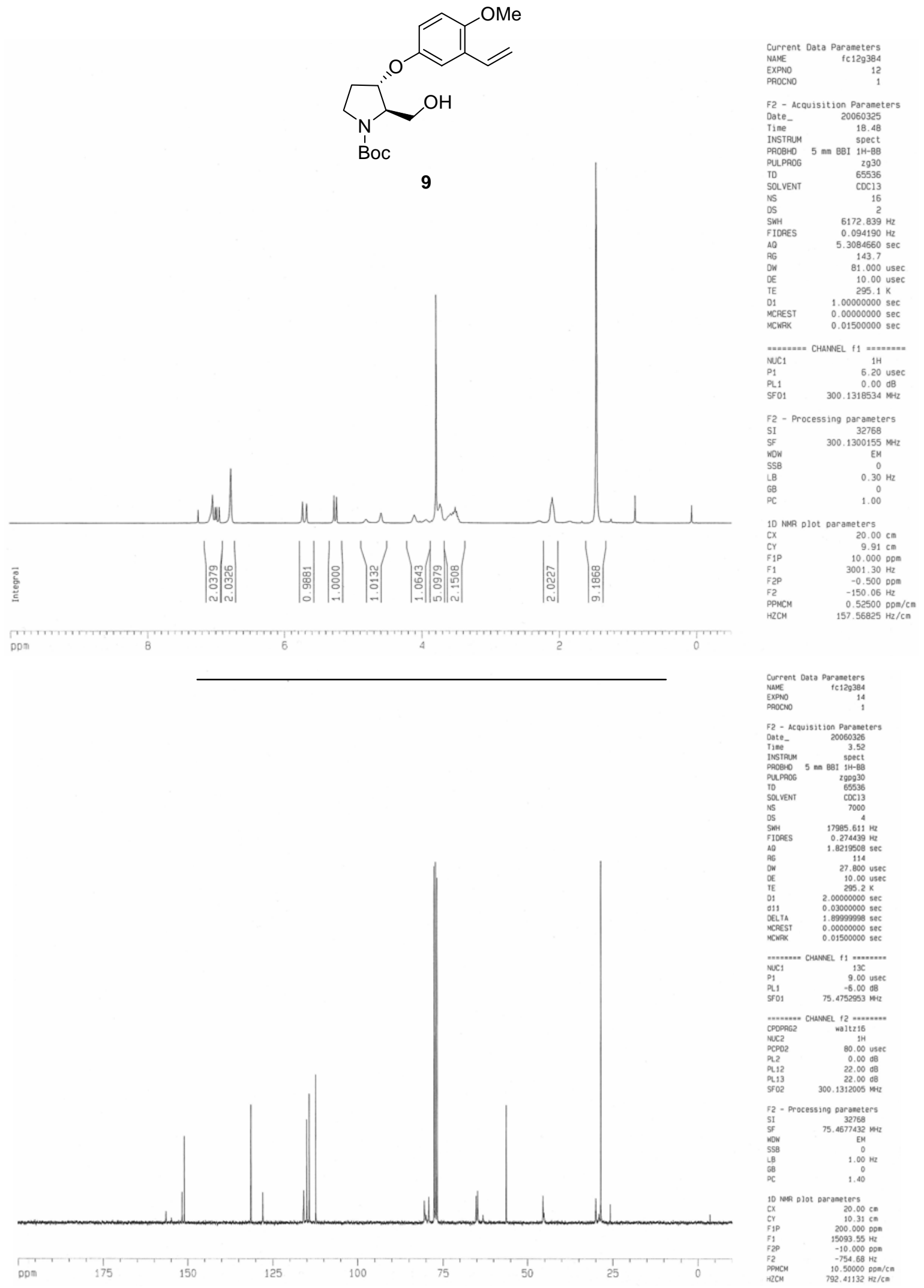


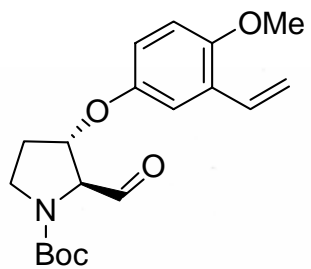

10

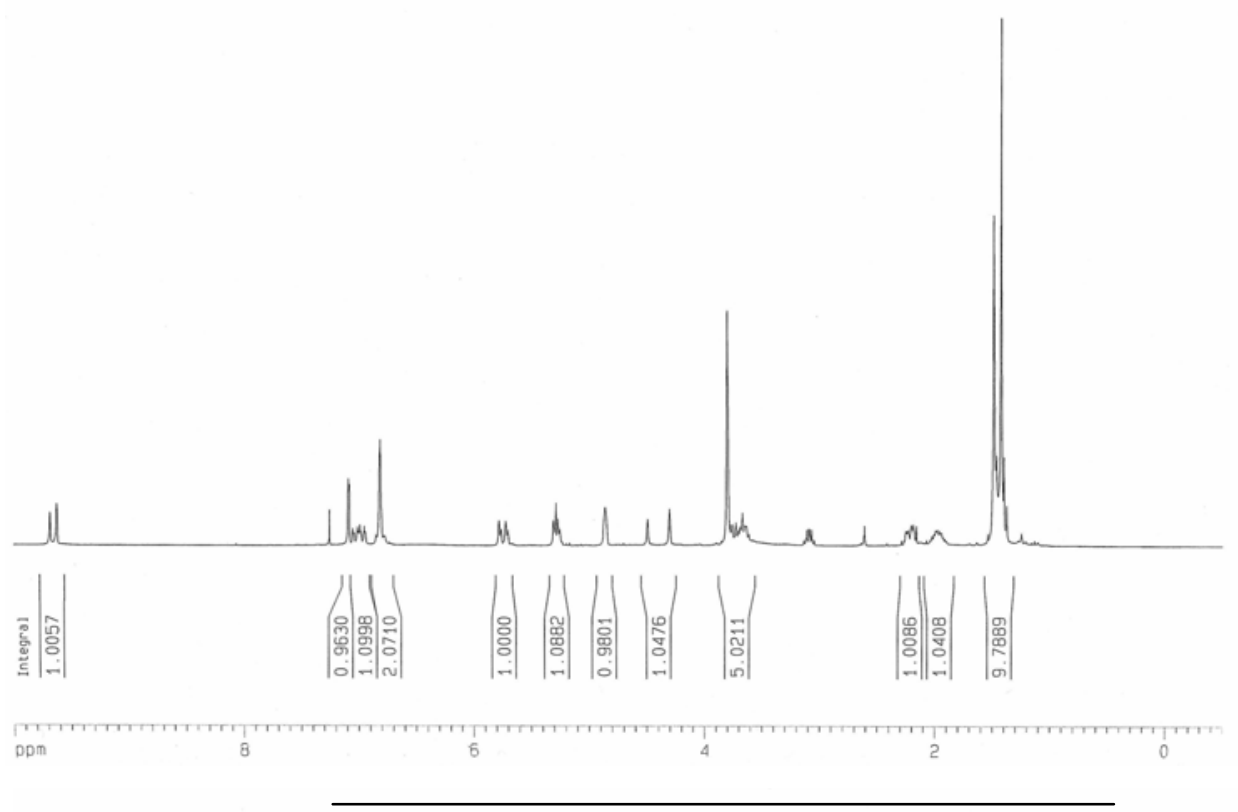

$\begin{array}{lr}\text { Current Data Parameters } \\ \text { NANE } & \text { fC13t1858 } \\ \text { EXPNO } & 11 \\ \text { PROCNO } & 1\end{array}$

F2 - Acquisition Parameters

Date_ 20060403

Tine

РRОВНO $5 \mathrm{~mm}$ BBI $1 \mathrm{H}-\mathrm{HB}$

2930
PULPAOG
32789

TO

solvent

NS

SWH

DE

TE
01
MCREST

$294.9 \mathrm{~K}$
$1.0000000 \mathrm{sec}$

"....... CHAMNEL 11 .........

$\begin{array}{ll}N_{11} & 1 \mathrm{H} \\ \mathrm{P}_{1} & 6.20 \text { usec } \\ \mathrm{P}_{1} & 0.00 \mathrm{~dB}\end{array}$

SF $01 \quad 300.1328512 \mathrm{dBH}$

F2 - Processing paraneters

SI 32768

$\begin{array}{lr}\text { SF } & 300.1300149 \\ \text { NSB } & \text { EM } \\ \text { SSB } & 0\end{array}$

$\begin{array}{ll}\text { SSB } & 0 \\ \text { LB } & 0.10 \mathrm{~Hz} \\ \mathrm{~GB} & 0 \\ \mathrm{PC} & 1.00\end{array}$

10 NMP plot paraneters

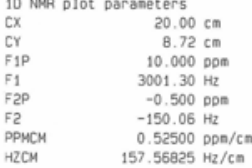

Current Data Parameters
nuve $\quad$ fc13t185B
EXPNO

PPOCMO

F2 - Acquisition Paraneters

Date-
Tine
Ther

19.15

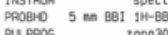

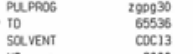

$\begin{array}{ll}\text { SQ VQVENT } & \\ 15 & 2000 \\ \text { OS } & \end{array}$

$\begin{array}{ll}\text { SWH } & 17985.611 \mathrm{~Hz} \\ \text { Fiofes } & 0.274439 \mathrm{~Hz}\end{array}$

$1,8219508 \mathrm{~B}$

$\begin{array}{rr}27 & 128 \\ \text { On } & 27.800 \text { usec } \\ \text { of } & 10.00 \text { usec }\end{array}$

$2005.0 \mathrm{~K}$

$2.00000000 \mathrm{sec}$
011 $\quad 0.03000000 \mathrm{sec}$

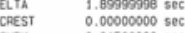

$0.01500000 \mathrm{sec}$

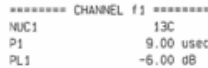

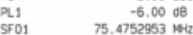

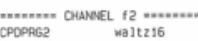

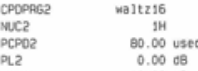

$\begin{array}{ll}\text { PL2 } & 0.0000 \\ \text { PL12 } & 22.0008\end{array}$

SF02 300.1312005

E2 - Processing paraneters

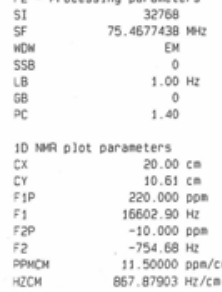

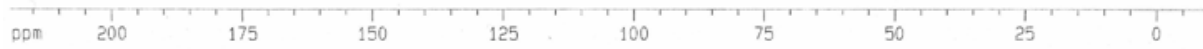




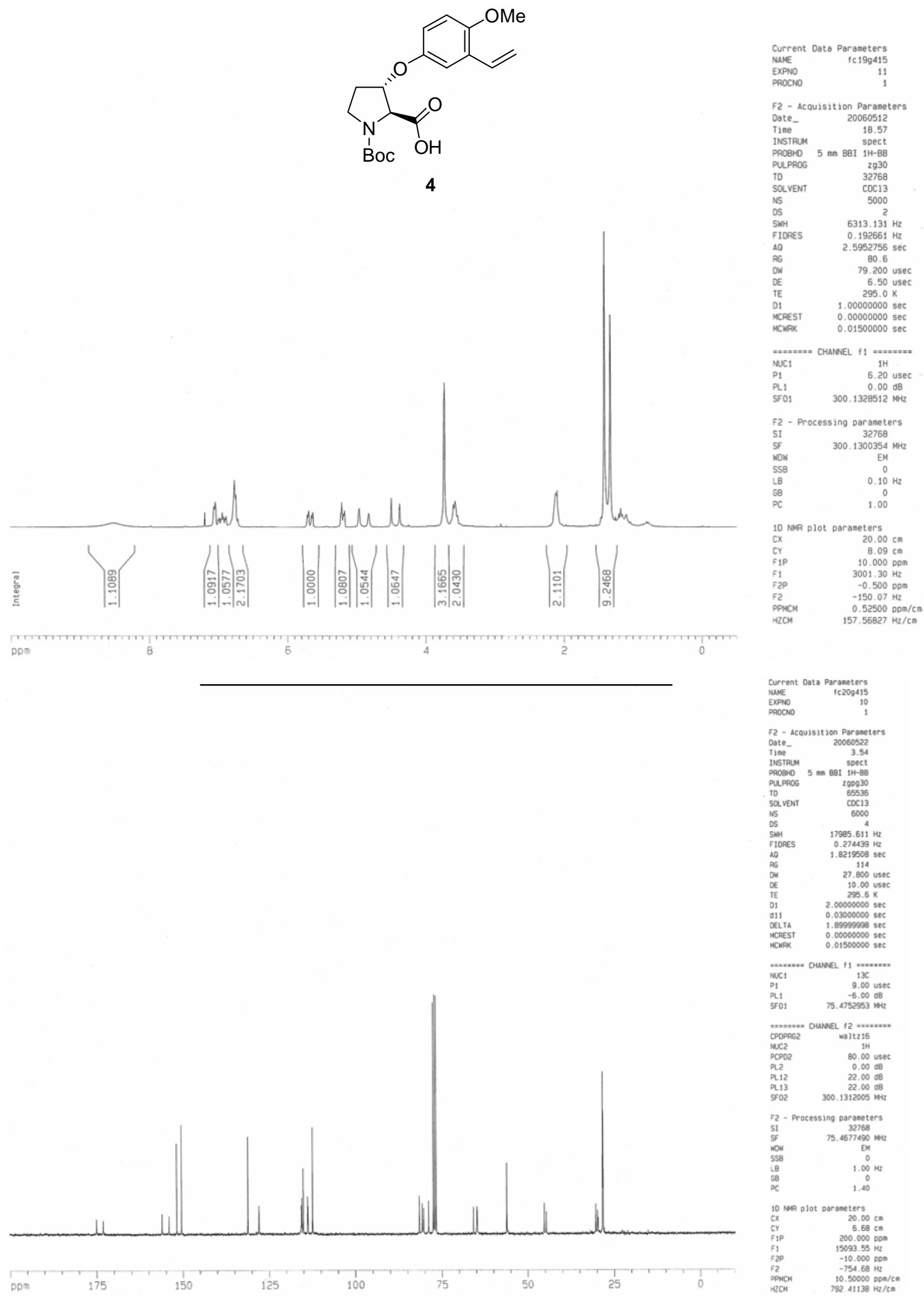




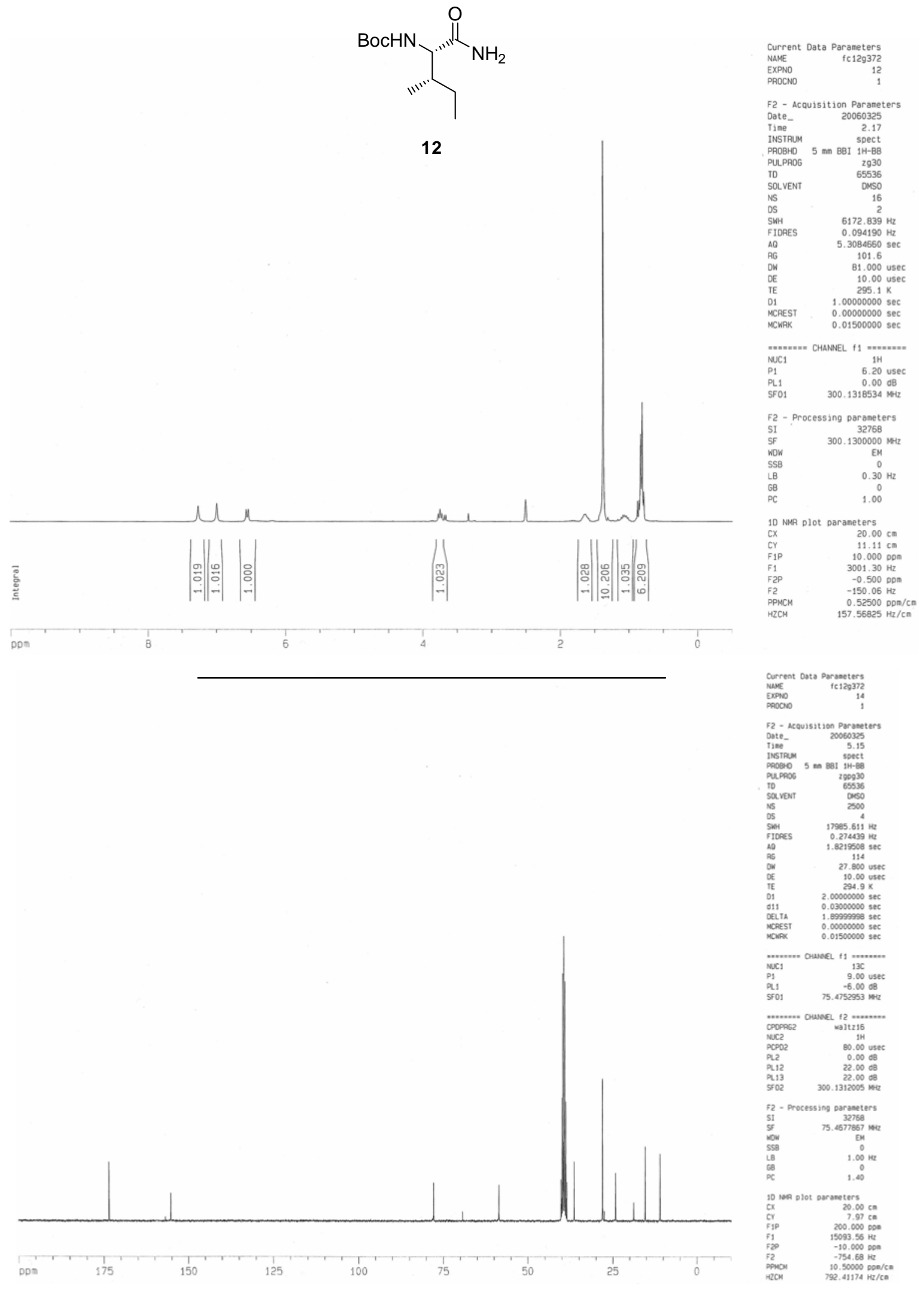



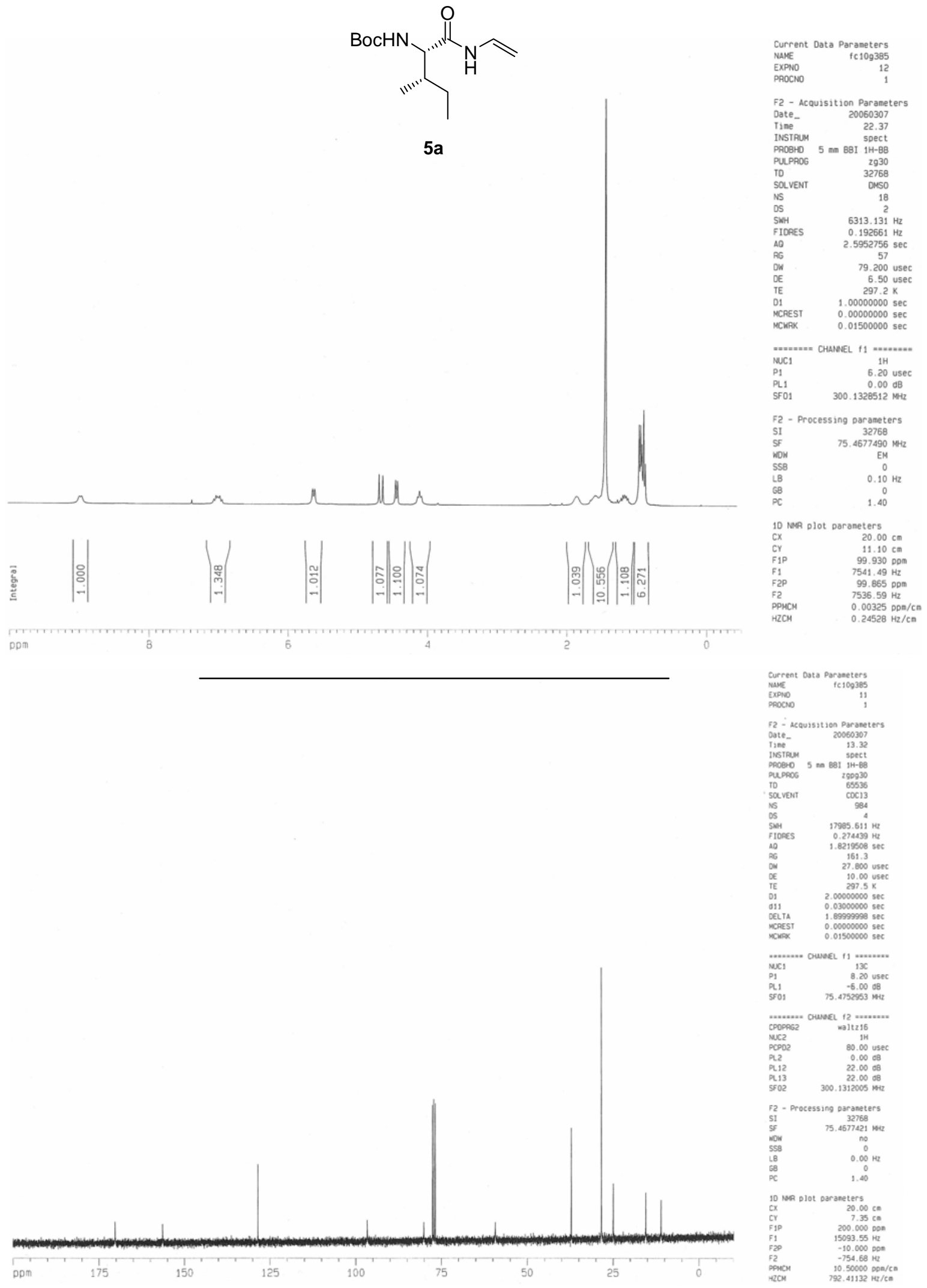

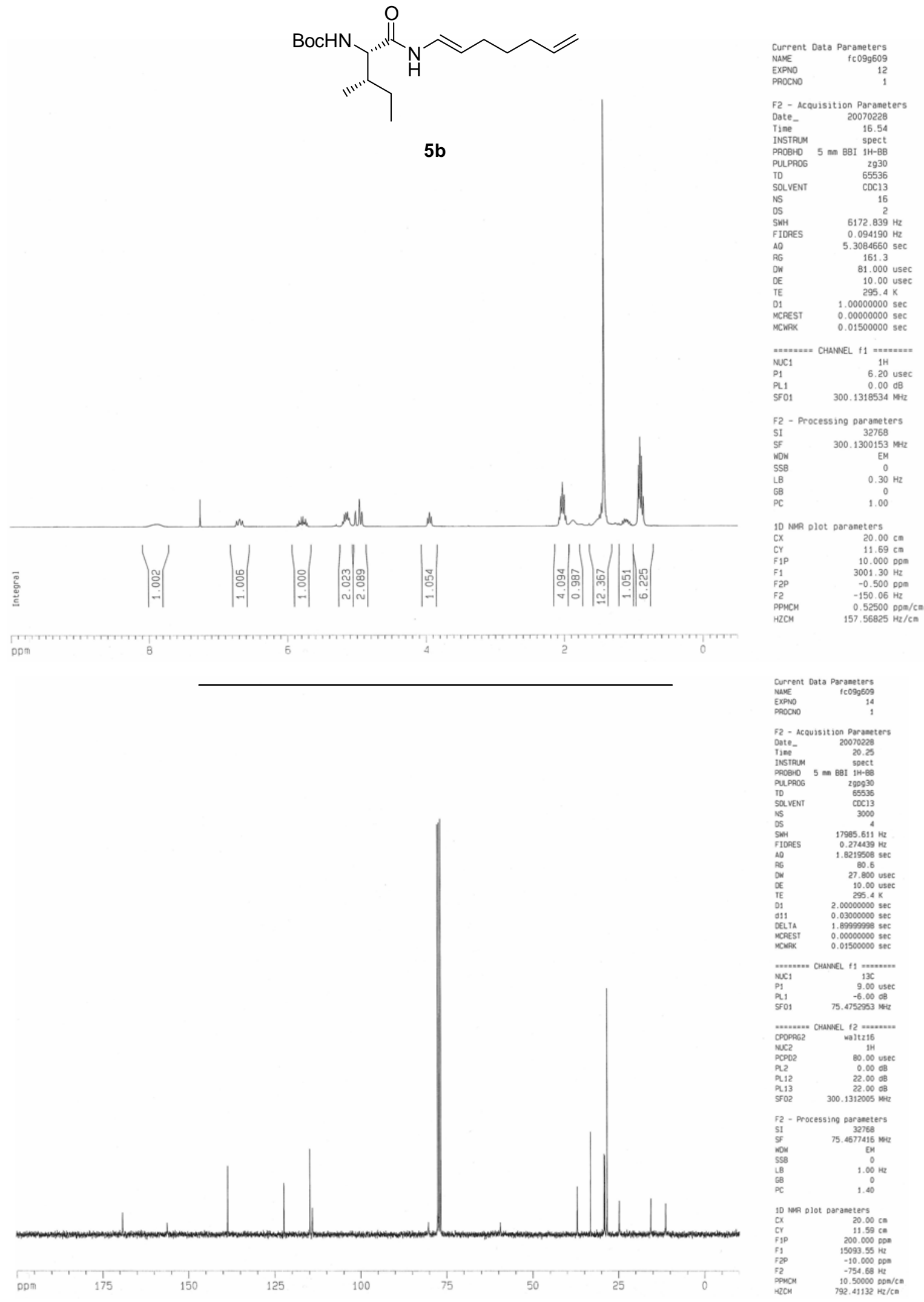


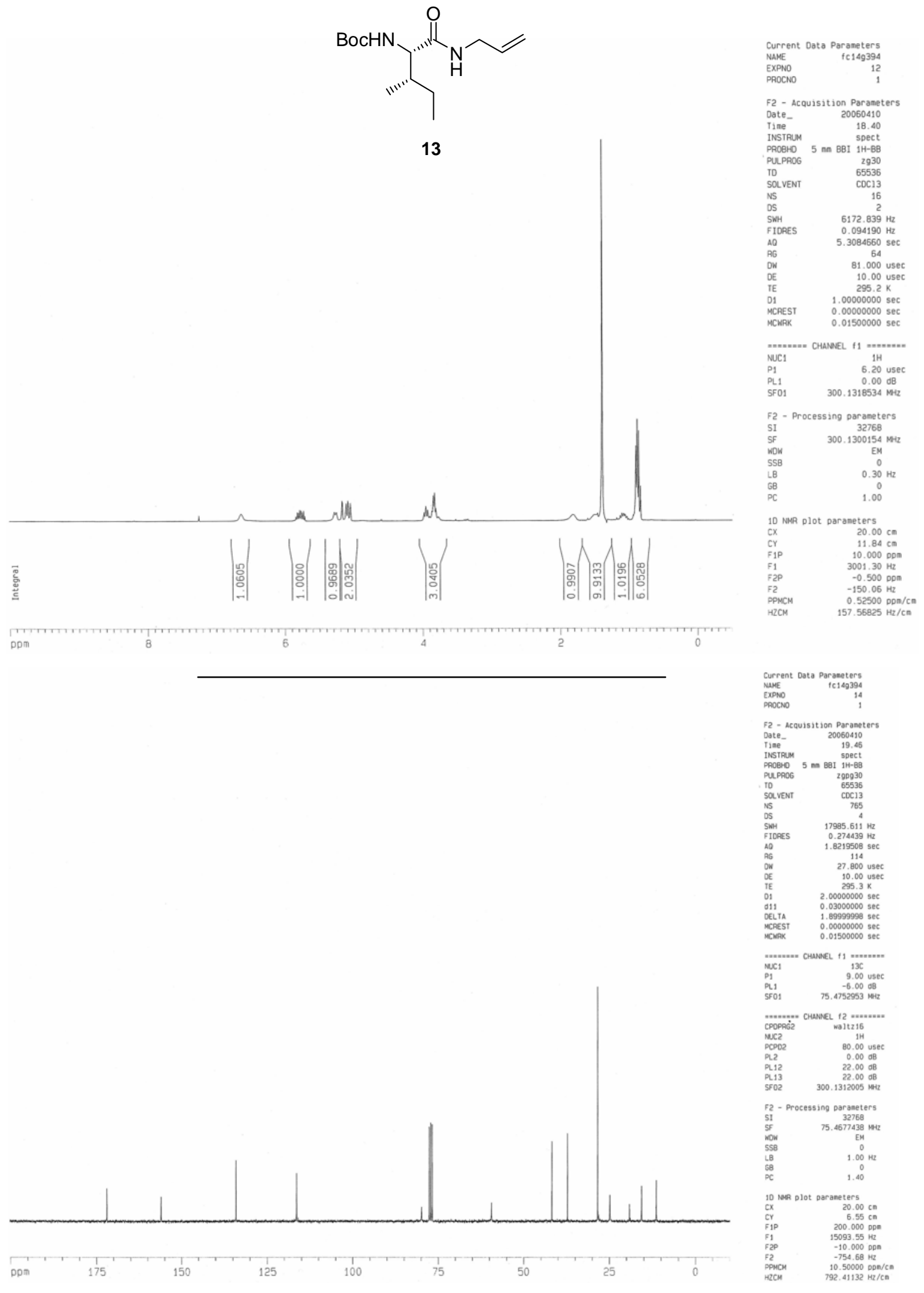




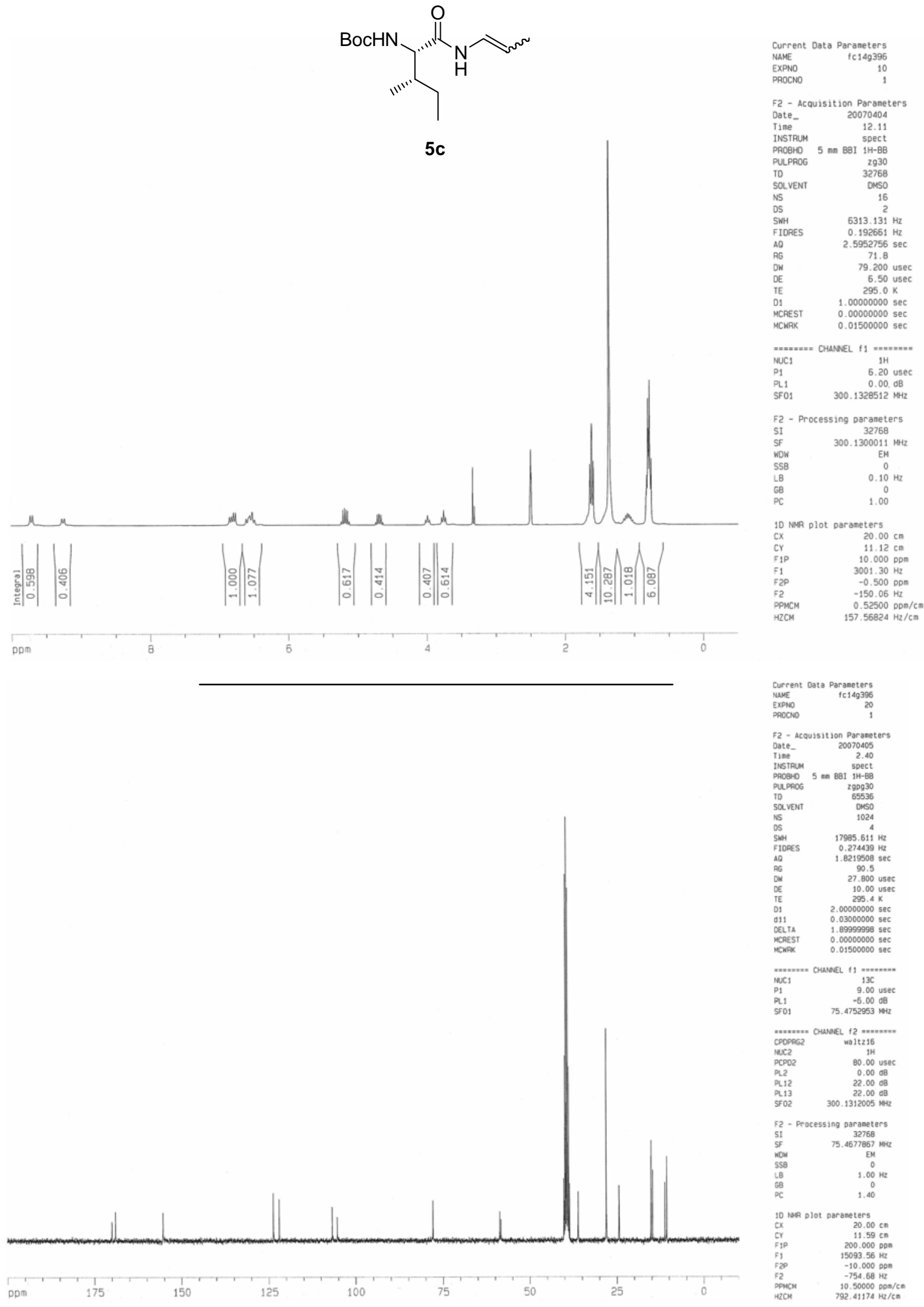



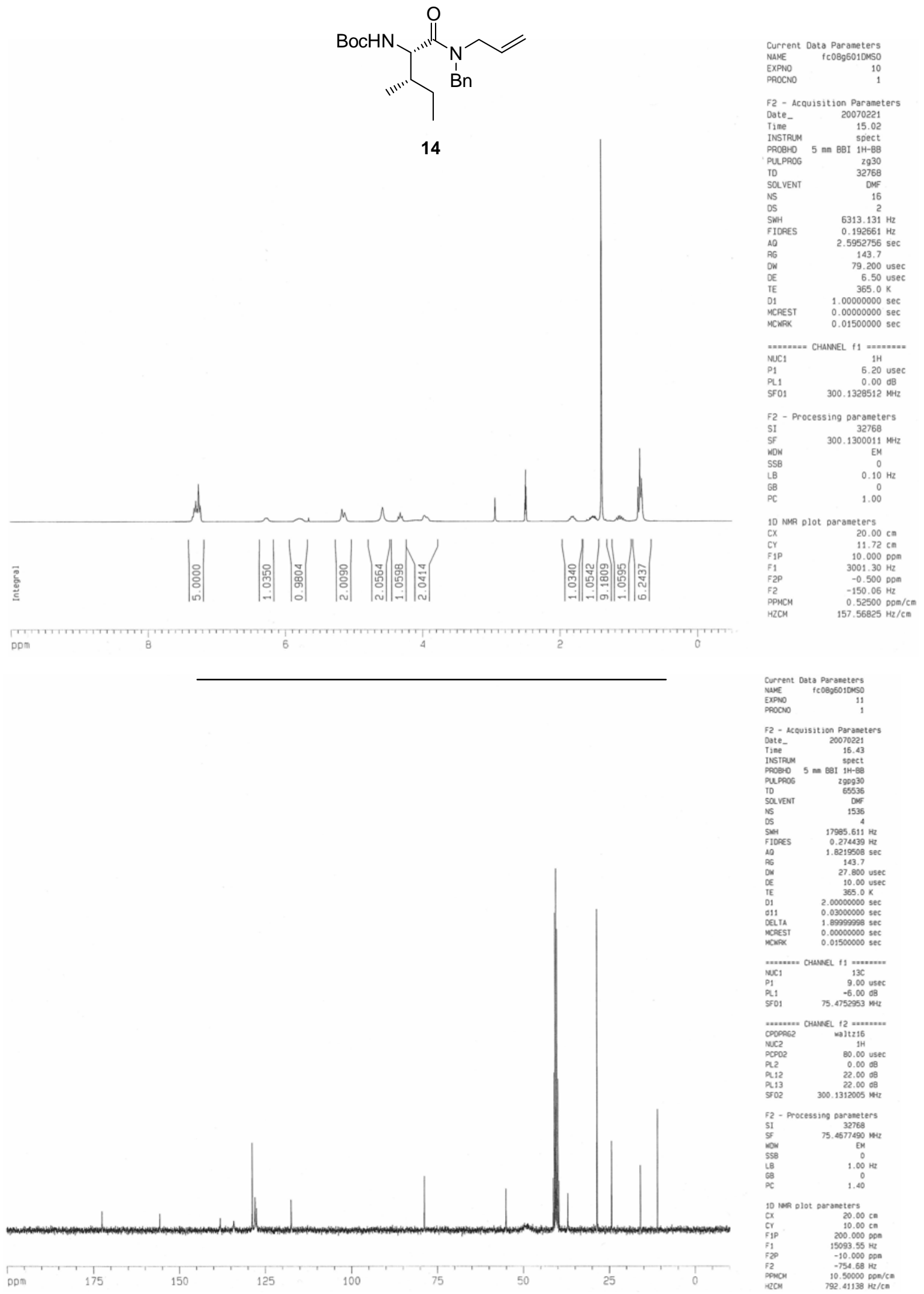


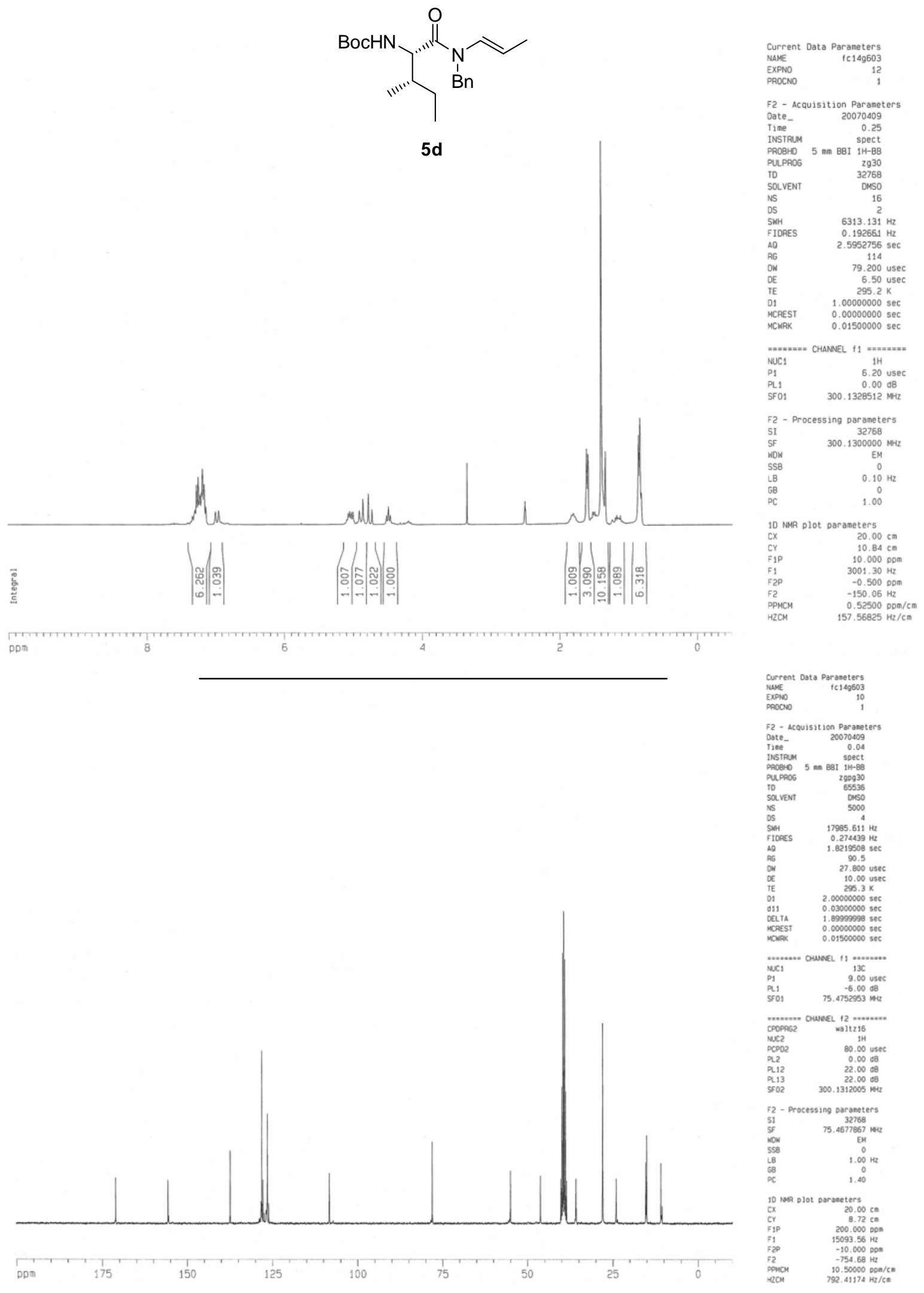



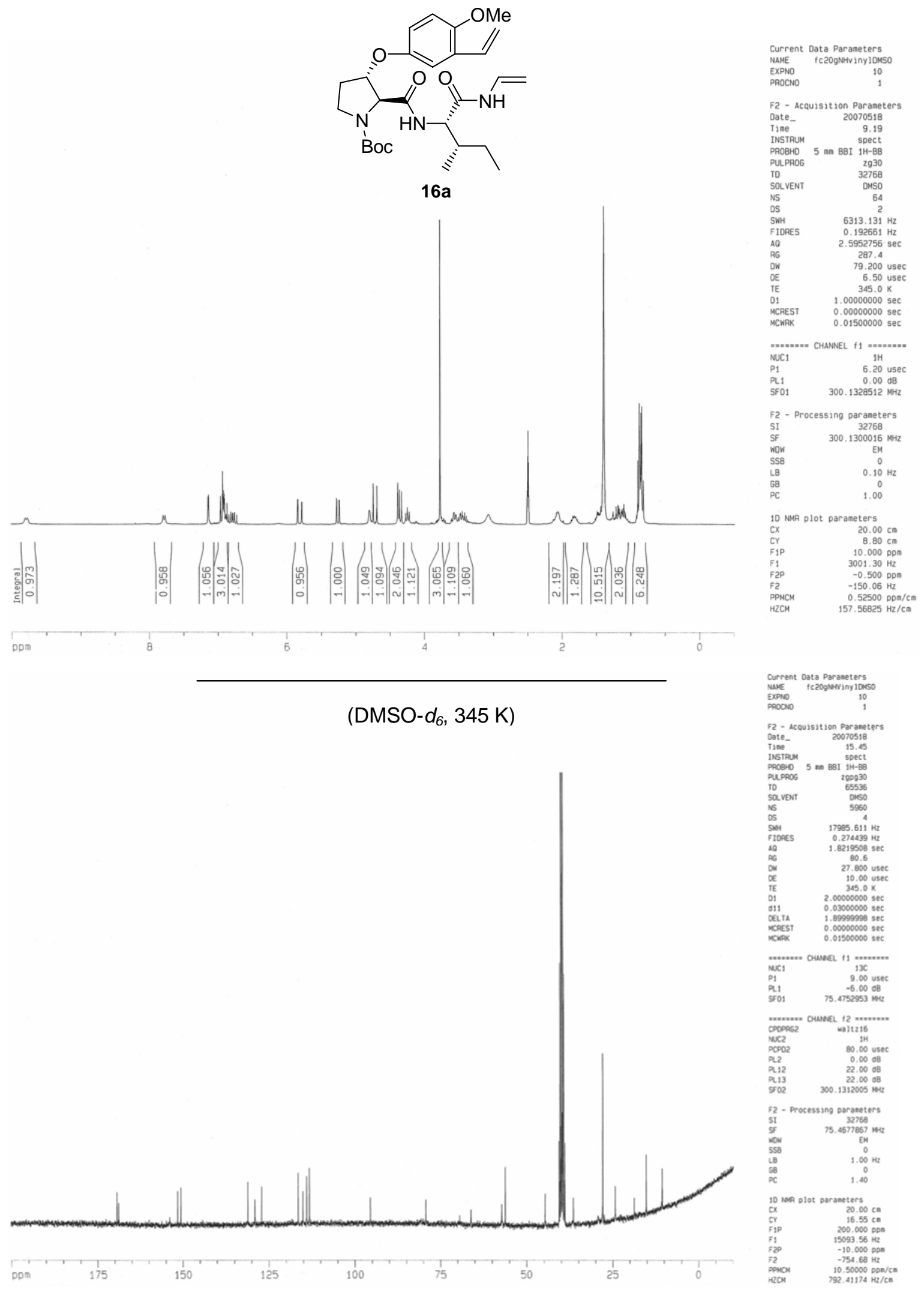


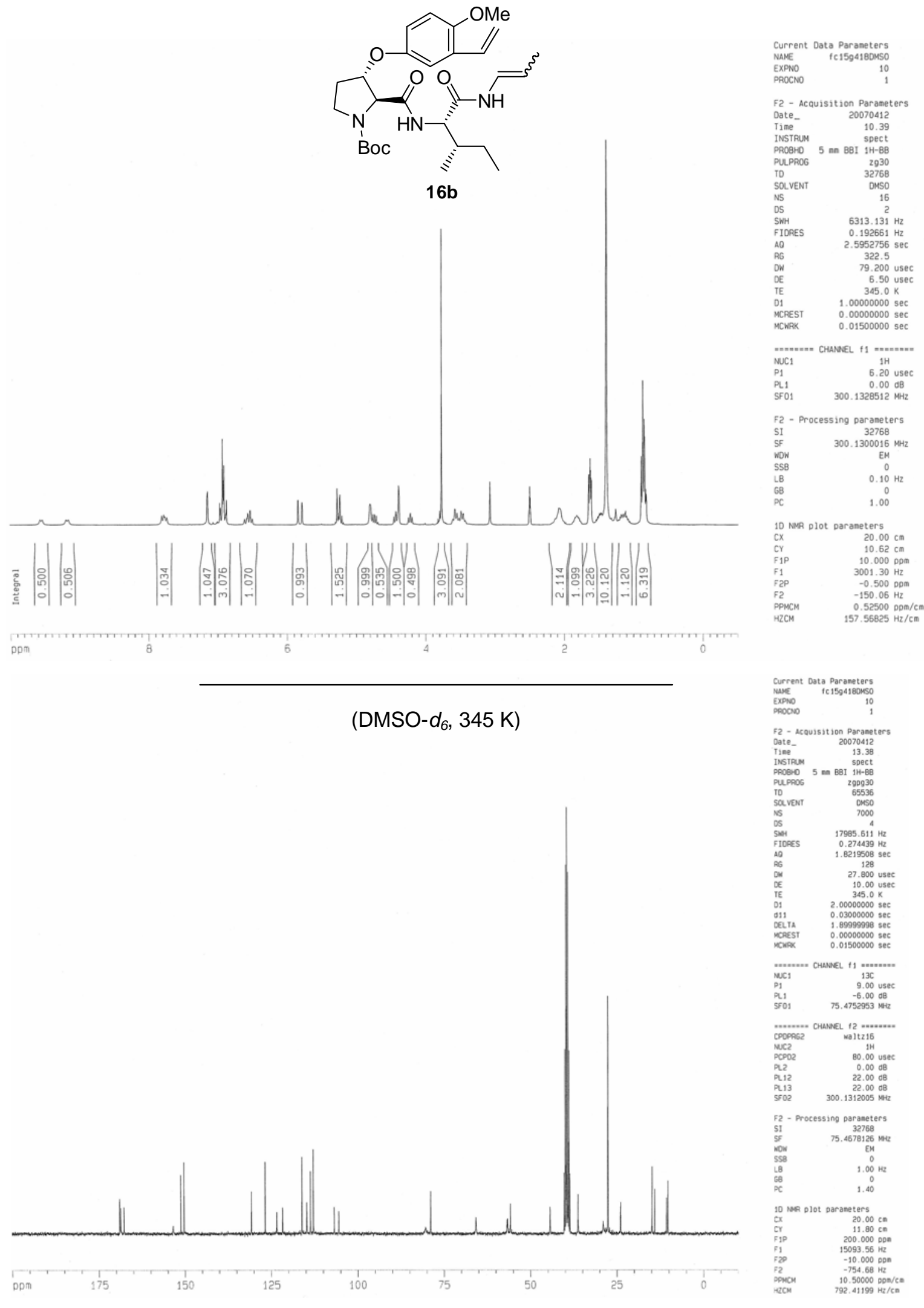




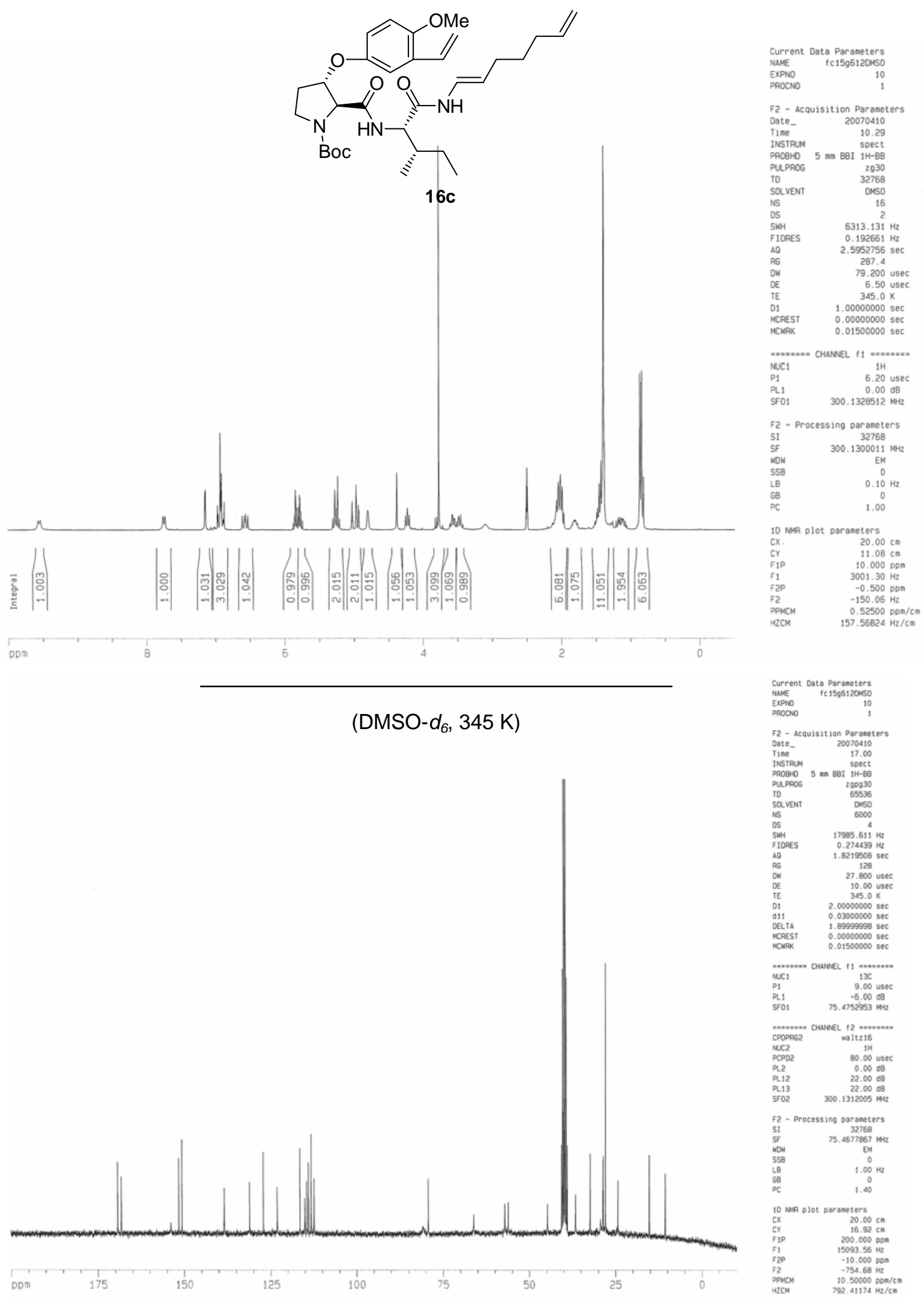




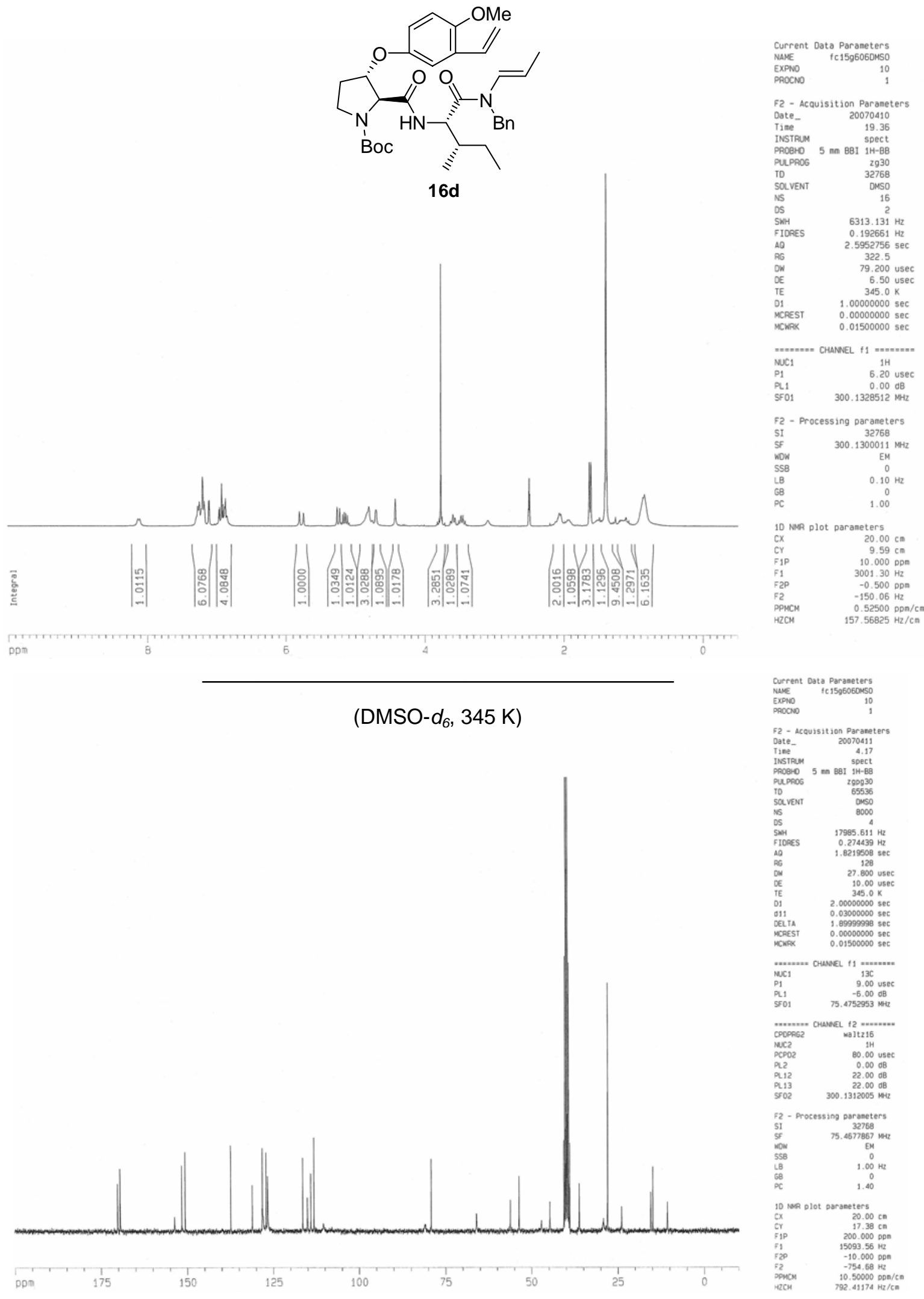




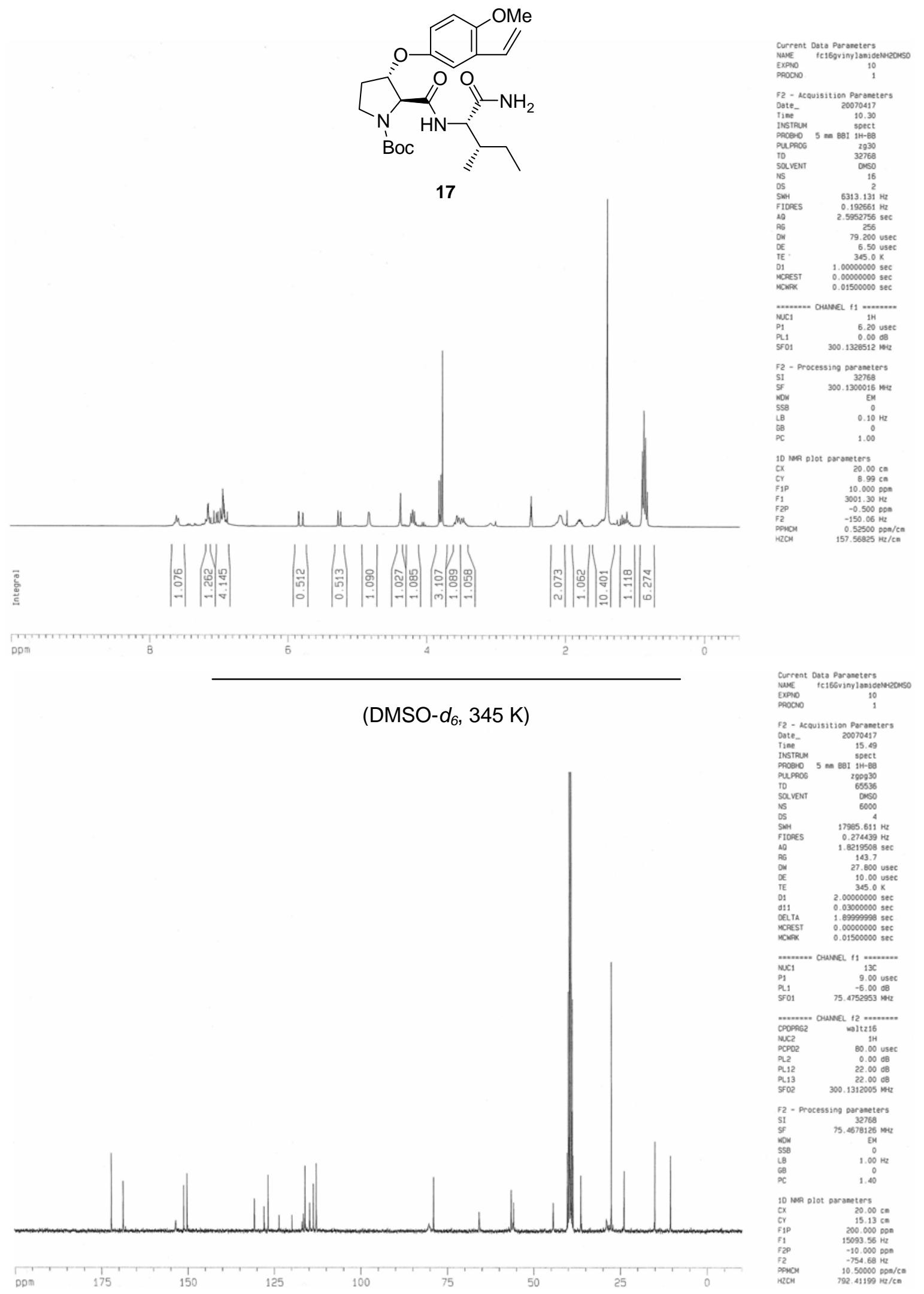




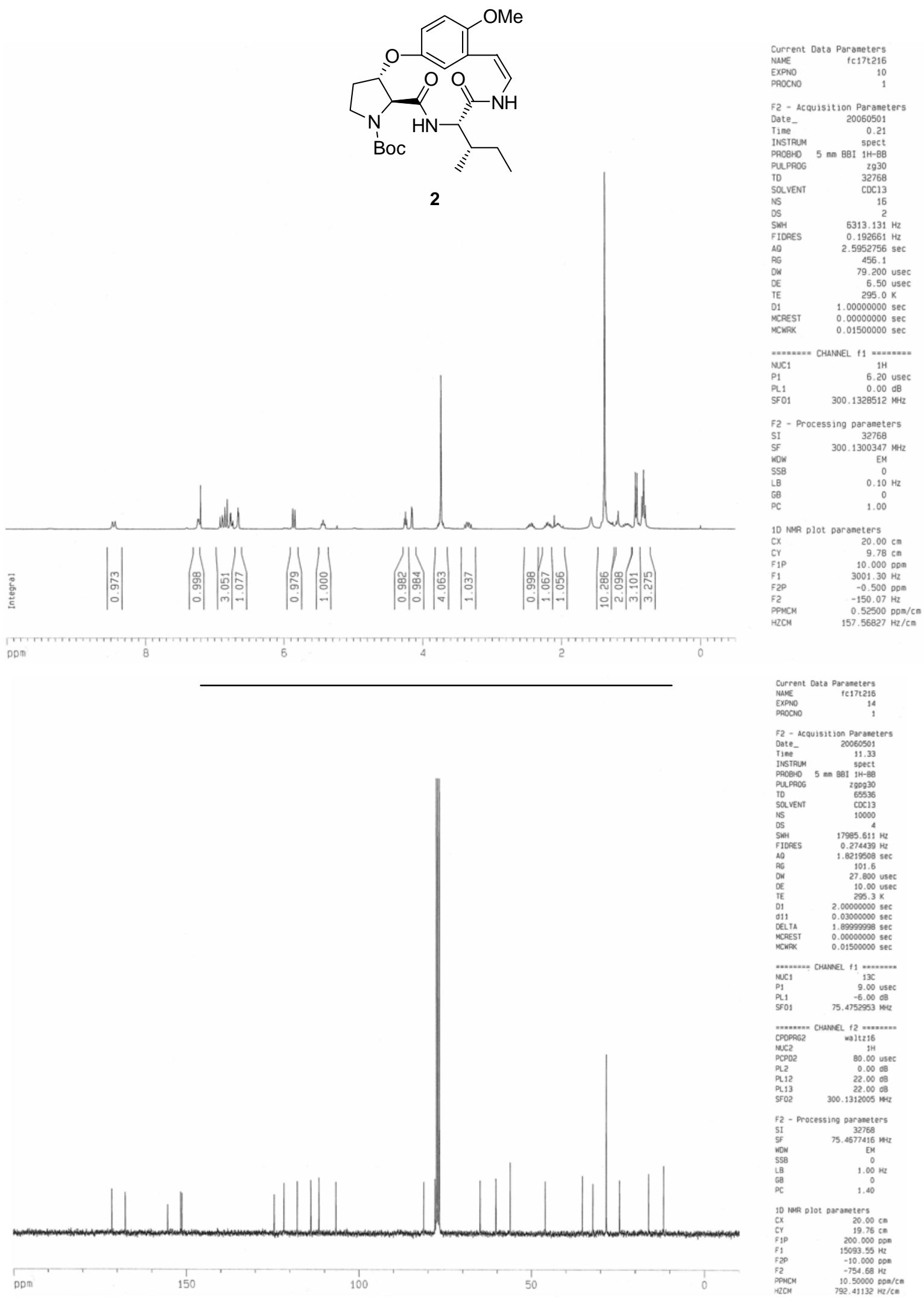




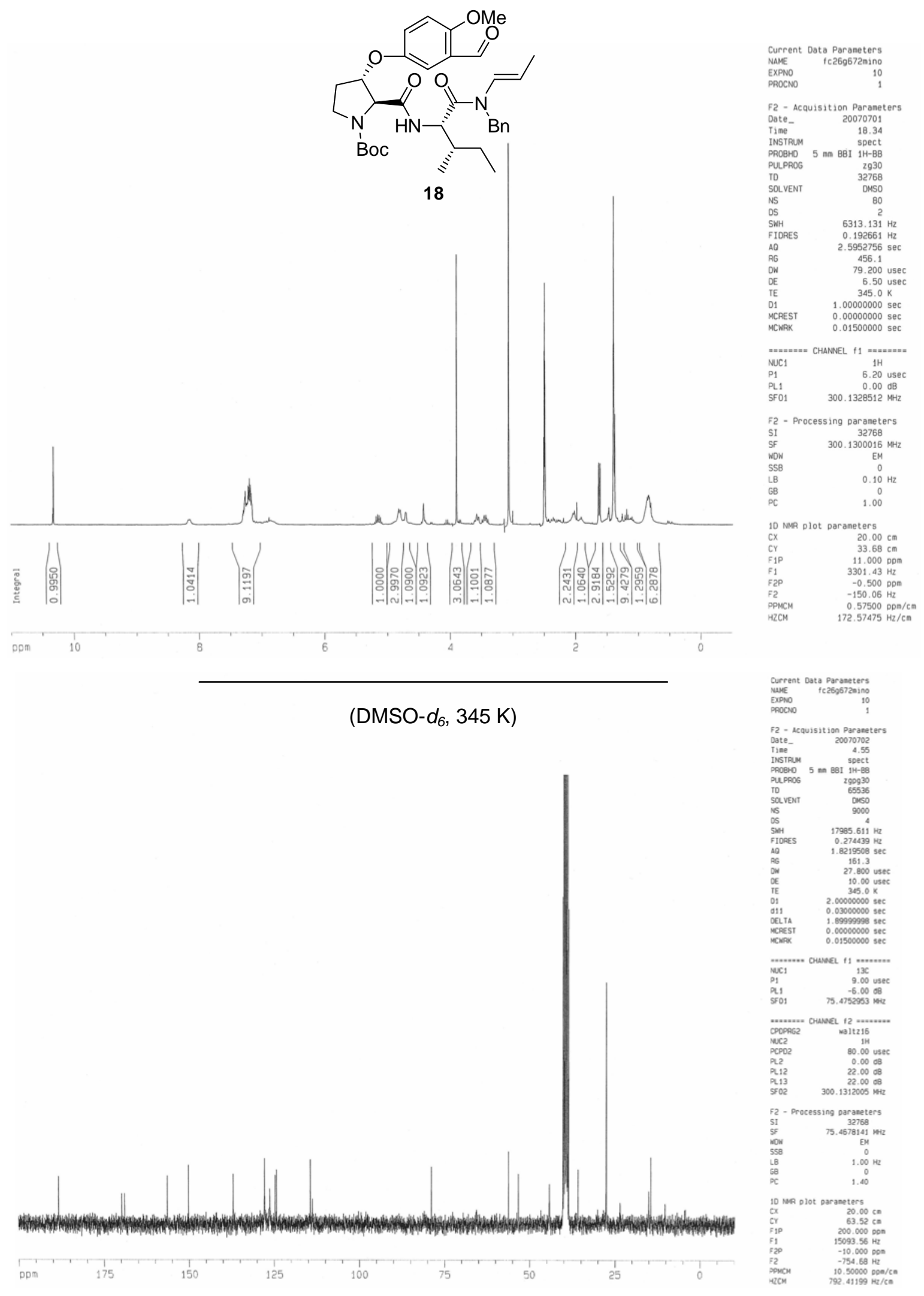




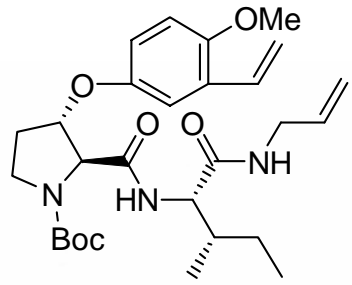

20

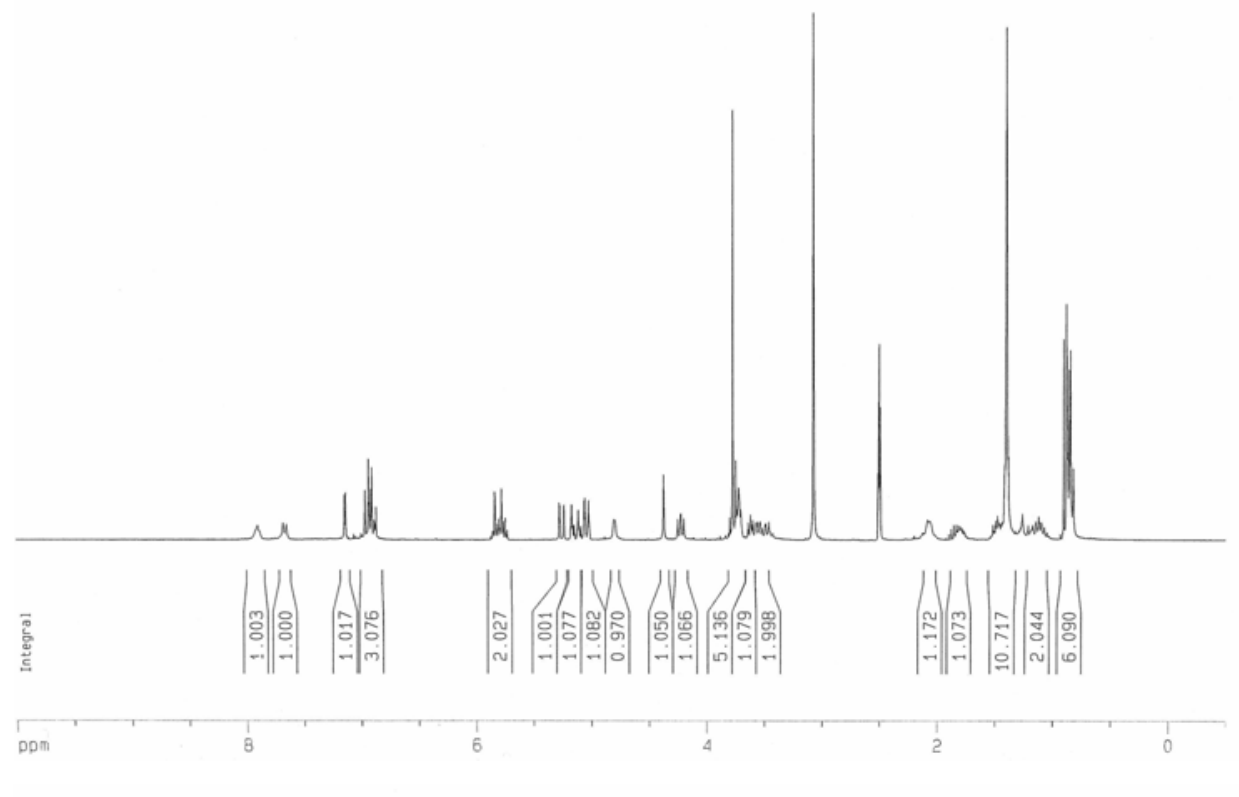

$\left(\mathrm{DMSO}-d_{6}, 345 \mathrm{~K}\right)$

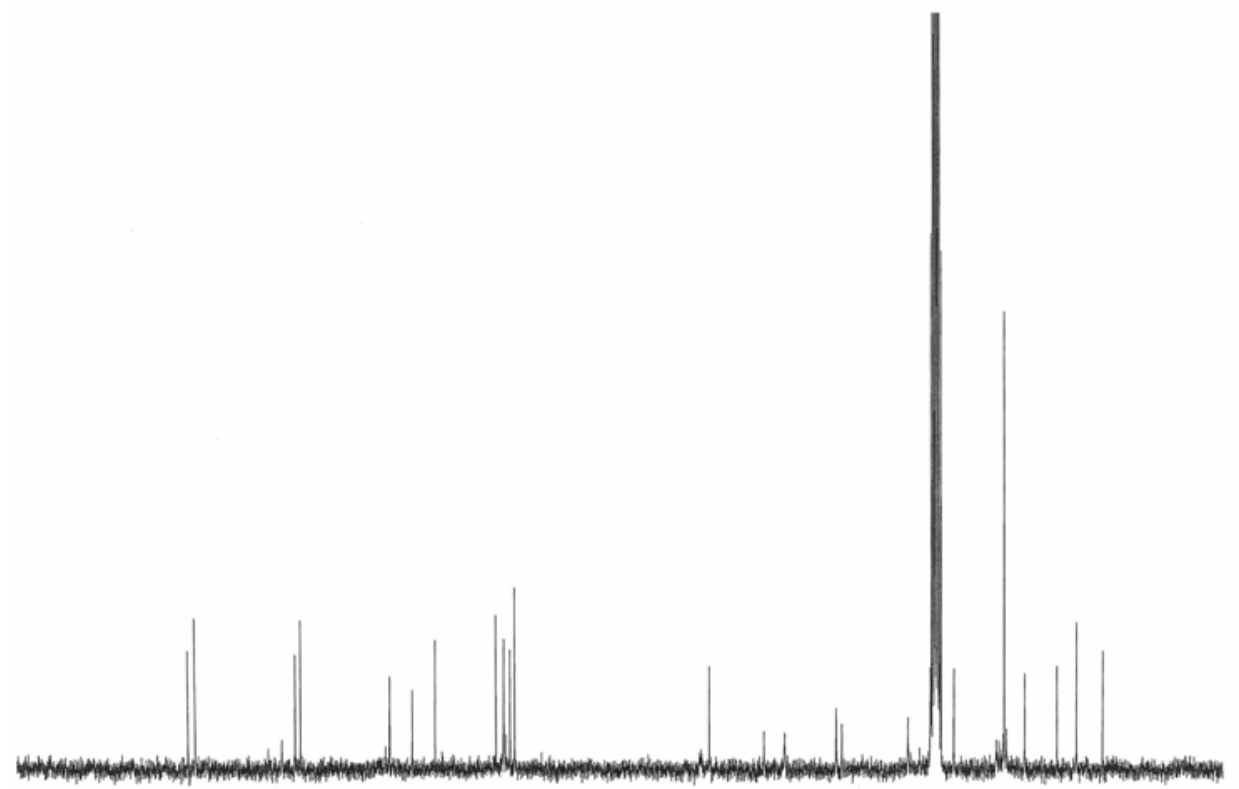

ppm

150

${ }_{100}^{1}$

50
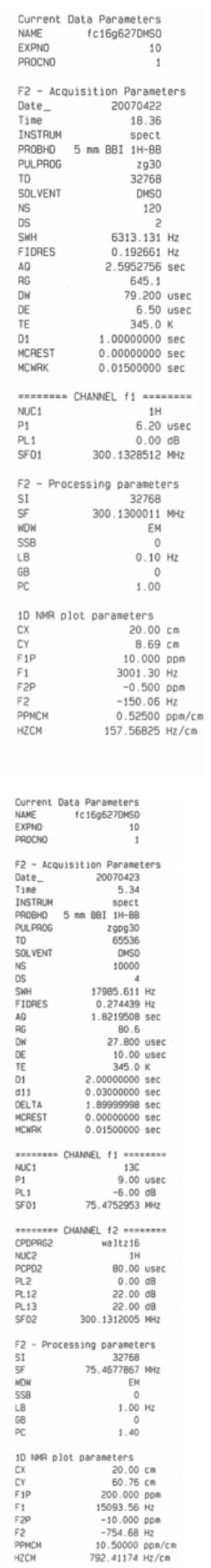


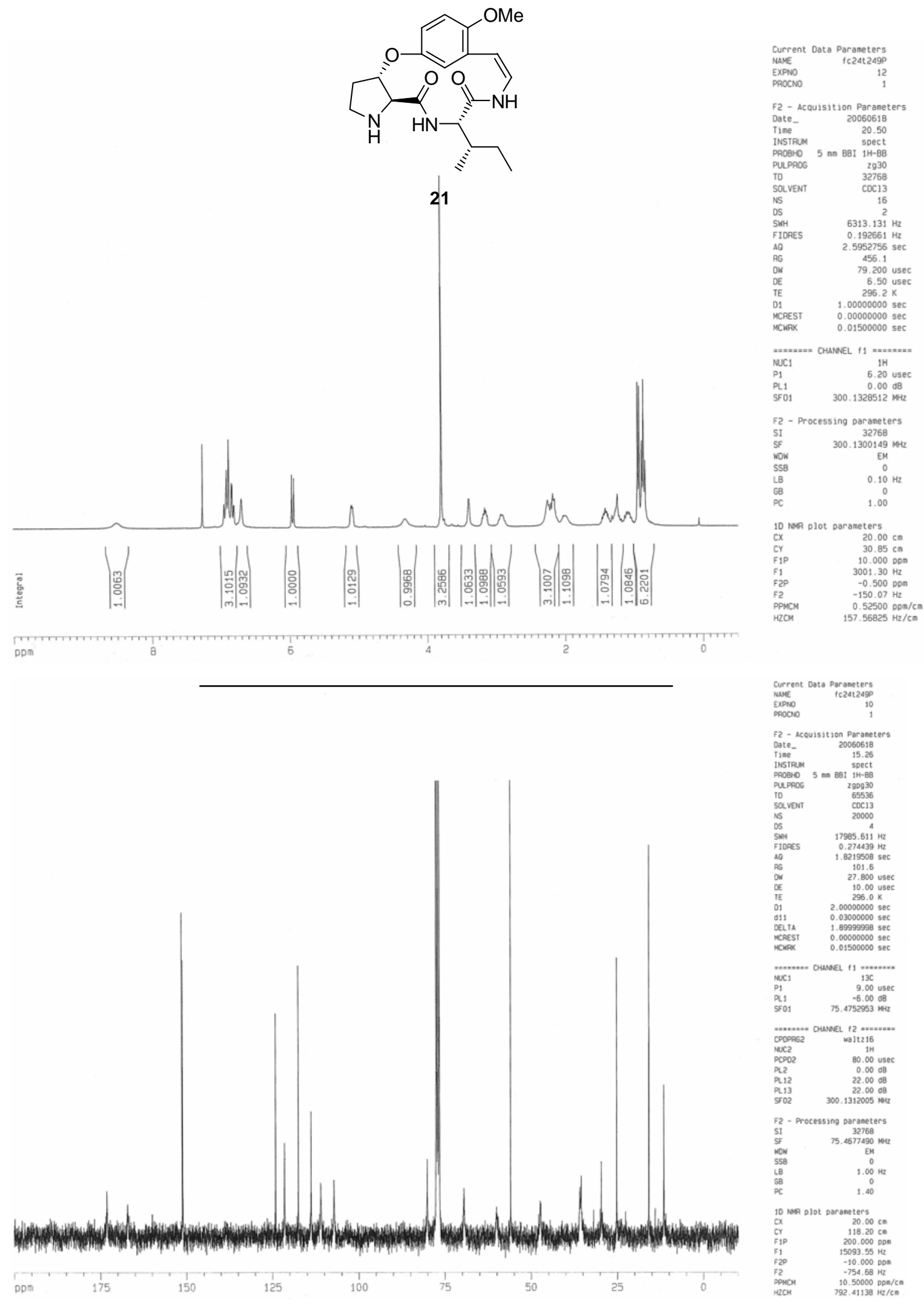


Supporting Information

\section{${ }^{1} \mathrm{H}$ and ${ }^{13} \mathrm{C}$ NMR spectra of synthetic paliurine $\mathrm{E}$}



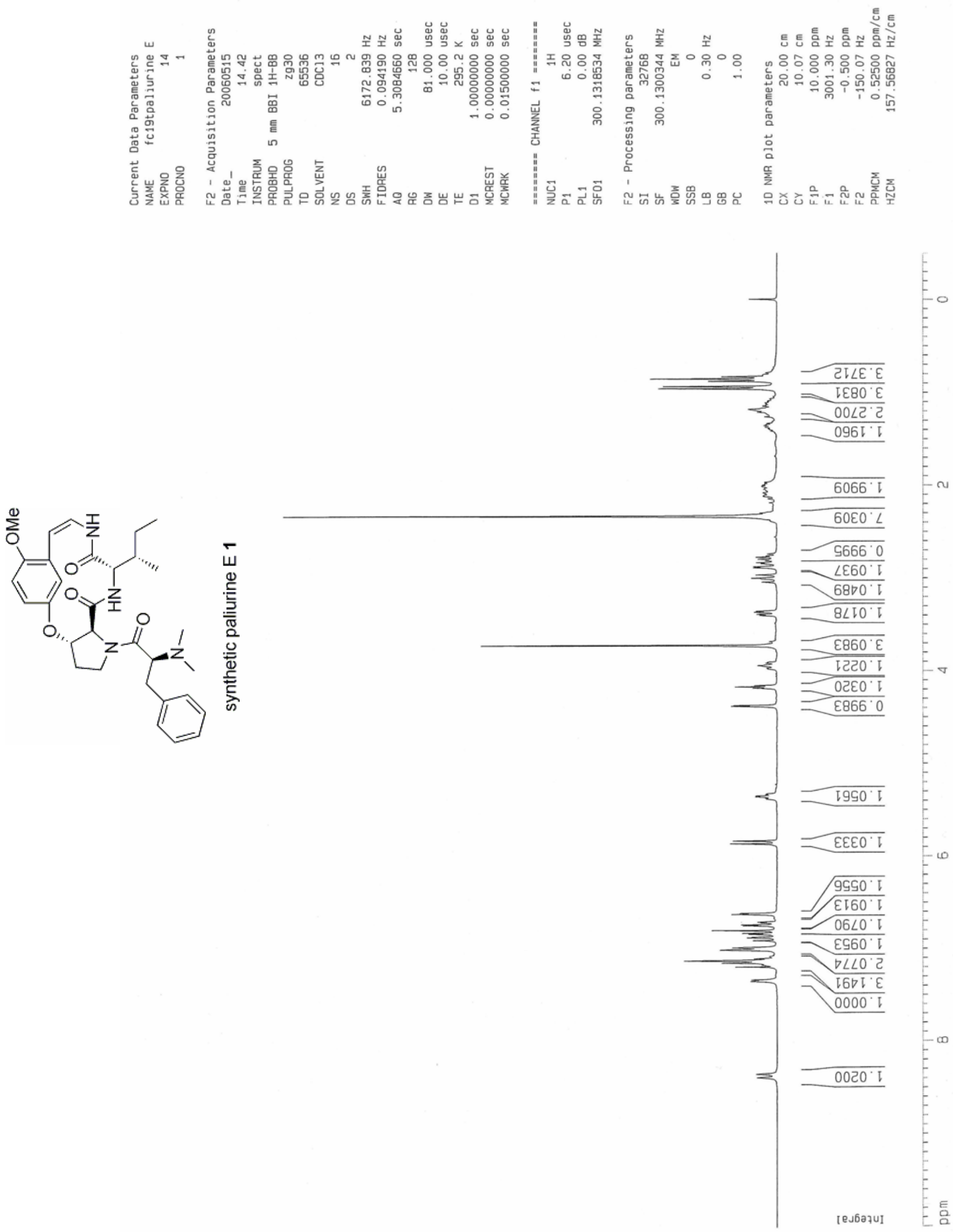

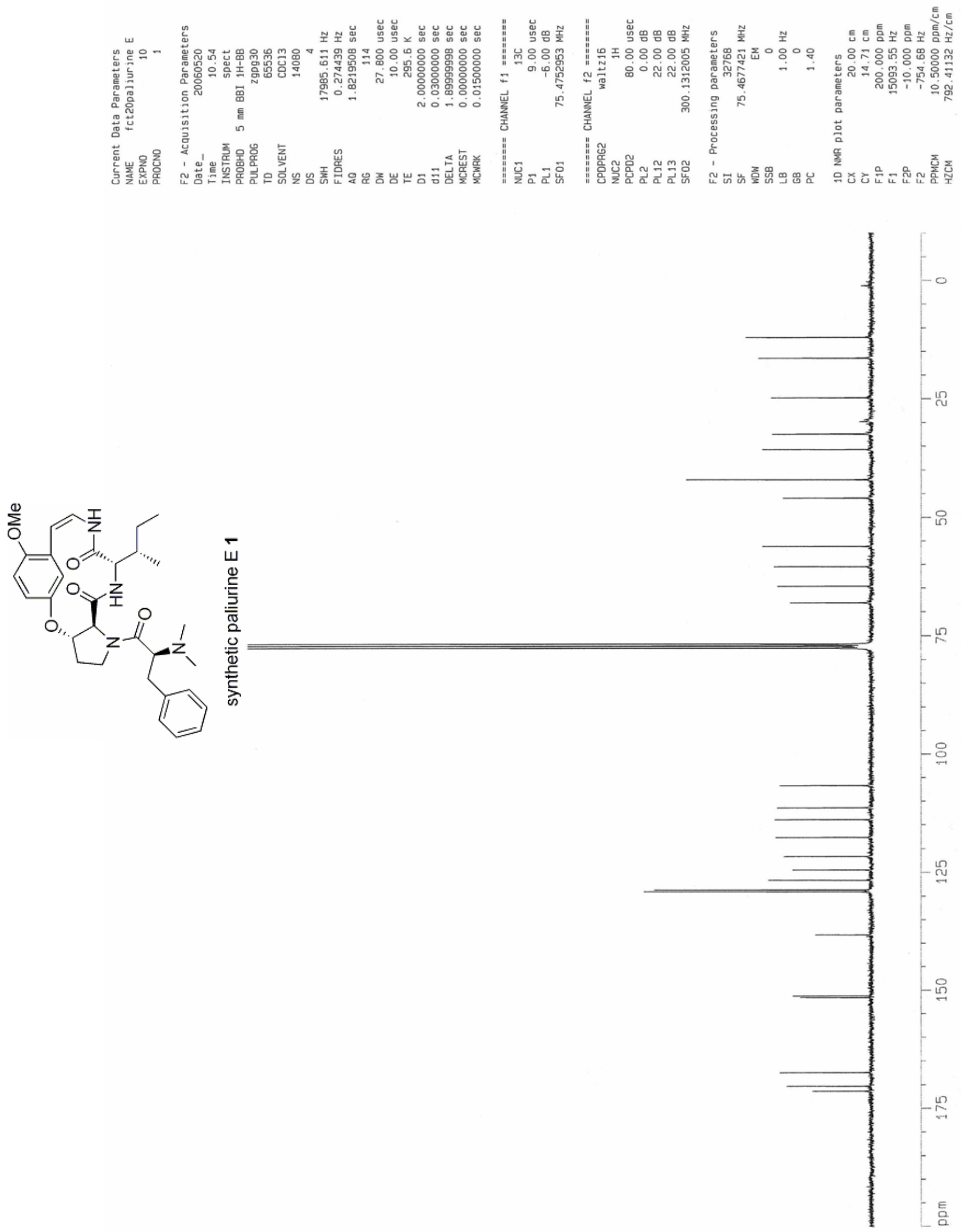